\title{
Ant Colony Algorithm Based Vehicle Routing Optimization of Tobacco Distribution
}

\author{
Qingsheng Shi ${ }^{1}$, Hongchun $\mathrm{Hu}^{2, *}$ and ${\mathrm{Ke} \mathrm{Lu}^{1}}^{1}$ \\ ${ }^{1}$ College of Electrical Engineering, Henan University of technology, Zhengzhou 450001, China \\ ${ }^{2}$ Staff Development Institute of China, Nation Tobacco Corporation, Zhengzhou 450003, China \\ ${ }^{*}$ Corresponding author
}

\begin{abstract}
Tobacco distribution Reducing energy consumption in tobacco logistics distribution process is one of the important measurements for energy saving and emission reduction of tobacco industry. Tobacco distribution vehicle routing optimization problem is essentially a typical NP problem. As a novel swarm intelligence optimization algorithm, Ant Colony Algorithm is adopted to solve the problem in the paper. First, a mathematical description of tobacco vehicle distribution problem is analyzed. And then, the basic principle of ACA is introduced followed by steps for tobacco logistics distribution vehicle routing using ACA. Finally, taking certain distribution network of Zhengzhou city for example, compared to simulated annealing algorithm, simulation experiments are carried out using an A CA procedure which is developed by matlab m-language to solve tobacco optimal distribution. The results show that it is feasible to solve Tobacco optimal distribution problem using ACA.
\end{abstract}

Keywords-tobacco distribution; routing optimization; ant colony algorithm

\section{INTRODUCTION}

At present, the domestic tobacco companies is mainly based on the experience to choose the path of tobacco distribution vehicles, which often lead to the delivery of vehicles can not achieve the effect of short path [1]. Due to the rising cost of oil and labor, tobacco distribution costs have exceeded the cost of inventory. As one of the most important part of the tobacco distribution system, tobacco distribution vehicle routing optimization is very important to the whole tobacco distribution cost, benefit and speed. Therefore, how to choose the best tobacco distribution vehicle routing and timely deliver tobaccos to customers, becoming a hot issue in the field of tobacco research [2-3]. Vehicle routing optimization of tobacco distribution problem is essentially a kind of NP problem, which is difficult to solve by using exact optimization algorithm. Because of the advantages of parallel and fast solving, ant colony algorithm has been widely used in the field of rail transport, public transport scheduling, logistics and so on. However, the application of tobacco distribution is still relatively small[4]. Therefore, ant colony algorithm is adopted to solve tobacco distribution vehicle routing optimization problem in order to provide reference for the application and.

\section{TOBACCO DistribUtion VEHICLE ROUTING OPTIMIZATION MATHEMATICAL MODELING}

Tobacco distribution vehicle routing optimization can be described as: from the tobacco company distribution center with many cars more businesses to delivery, each customer's location and demand is known, each car load, reasonable arrangement of the requirements of automotive distribution route, total distribution distance is the shortest, also meet the following conditions:

1) all the distribution of vehicles to the distribution center as a starting point and eventually return to the distribution center;

2) every business only by a car to visit once, each car can only serve a line;

3) and no more than the maximum value of the vehicle load on each distribution path;

4) the route of each vehicle can not be repeated.

In summary, tobacco distribution vehicle routing optimization objective is to find the optimal tobacco distribution route according to the distribution task, so that the distribution cost is the lowest. Located in the distribution center has a $M$ car, the amount of each vehicle is $G_{k}(\mathrm{k}=, 1,2 \ldots, M)$ the maximum distance of a distribution of $D_{k}$, the need to $N$ customer shipping, customer $i$ tobacco demand for $g_{i}$, distribution center to the customer $i$ distance of $d_{0 i}$, the distance between the customer $i$ and $j$ is $d_{i j},(i, j=1,2, \ldots, N)$. And $n_{k}$ for the $k$-th car of distribution vehicle number (if $n_{k}=0$ means the kth car is not used); Set $R_{k}$ to the $k$ line, the elements of which $r_{k i}$ means the order of customer $r_{k i}$ in path $k$; let $r_{k 0}=0$ means the distribution center. The mathematical model of Tobacco distribution vehicle routing optimization problem can be expressed as:

$$
\min F=\sum_{k=1}^{M}\left[\sum_{i=1}^{n_{k}} d_{r_{k(i-1)} r_{k i}}+d_{r_{k n_{k}} r_{k 0}} * \operatorname{sign}\left(n_{k}\right)\right]
$$

$$
\sum_{\text {s.t. }}^{n_{k}} g_{r k} \leq G_{k}
$$




$$
\sum_{i=1}^{n_{k}} d_{r_{k(i-1)} r_{k i}}+d_{r_{k_{k}} r_{k 0}} * \operatorname{sign}\left(n_{k}\right) \leq D_{k}
$$

$$
0 \leq n_{k} \leq N
$$

$$
\sum_{k=1}^{M} n_{k}=N
$$

$$
R_{k}=\left\{r_{k i} \mid r_{k i} \in\{1,2, \cdots, N\}, i=1,2, \cdots, n_{k}\right\}
$$

$$
\operatorname{sign}\left(n_{k}\right)= \begin{cases}1, & n_{k} \geq 1 \\ 0, & \text { otherwise }\end{cases}
$$

\section{ANT COLONY Algorithm BASED TOBACCO Distribution VEHICLE ROUTING OPTIMIZATION STRATEgY}

\section{A. Preliminary of ACA}

ACA is a new kind of simulated evolutionary algorithm, which is proposed by Dorigo M, etc in 1991 based on the study of real ant colony collective behavior in the nature [5]. When the ant in biosphere search for food sources, it can release a specific ant secretions (pheromone) in the walked path to let other ants in certain range detect and influence their behavior. The more ants walk through certain path, the more the information hormone is, so as to the higher probability the ants choose the path, which add to the attraction of the path strength. Thanks to this internal bio-synergy mechanism, ants groups find a shortest route without awareness.

Combined with traveling salesman problem, Ant Colony Algorithm's implement process can be described as follows:

Given an $n$-city TSP problem and an ant colony with $m$ ants, let's see how to establish the model based Ant Colony Algorithm. Let $d_{i j}(i, j=1,2,3, \ldots, n)$ be distance between the he city $i$ and city $j$; $b_{i}(t)$ be the the number of ants in city $i$ at time $t$; $\tau_{i j}(t)$ be the trail degree of edge $(i, j)$ at time $t ; \eta_{i j}$ be the visibilty of edge $(i, j) ; \alpha$ be the information heuristic factor, reflecting the relative importance of trail; $\beta$ be relative importance of visibility; $P_{i j}^{k}(t)$ be transition probability of ant $k$ from the city $i$ to city $j$ at time $t$.

Assuming that, $m$ ants are placed into $n$ cities randomly, then each step of each ant's action is to choose a city which has not beed visited based on certain condition. At the same time, the remained $\tau_{i j}(t)$ on each path should be updated after one iteration. Based on the principle above, the condition to choose the next city is as follows: the initial $\tau_{i j}(t)$ is the same, when the ant start from city $i$, and select the next city to visit according to state transition rules. Eq.(8) gives out the transition probability by which the ants move from city $i$ to city $j$.

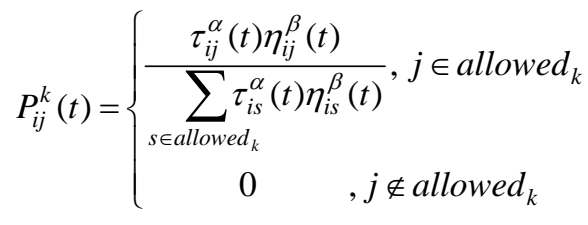

here, allowed $_{k}=\{1,2,3, \ldots, n\}$-tabu $u_{k}$ stand for all of the cities ant $k$ is allowed to visit in the next step; $t a b u_{k}$ is the table tabu, which records the cities ant $k$ has visited, it varies along with the evolution process of ants; $\mathrm{s}$ is the next city that ant $k$ is allowed to visit in the next time which is not in the table tabu. The state transition rules is as follows:

$$
j=\left(\begin{array}{c}
\arg \underset{j \in t a b u}{\operatorname{Max}}\left\{\left[\tau_{i j}(t)\right]^{\alpha}\left[\eta_{i j}(t)\right]^{\beta}\right\}, q<q_{0} \\
J
\end{array}\right.
$$

here, $q$ is a random variable uniformly distributed in [0,1], $q_{0}$ is a pre-given constant in $[0,1]$. If $q>q_{0}, J$ is the next city to select by the probability $P_{i j}^{k}$.

Ants select the cities to visit according to state transition rules mentioned above, and eventually find a closed path. If all the ants have completed their closed path, an iteration is finished. Then, the path trail should be updated using global information. And then, next iteration will start till the maximum number of iterations is reached.

To avoid heuristic information submerged by residual information contents due to excess of residual pheromone, it is necessary to update the residual information after one iteration. The path can be adjusted according to Eq. (10) and Eq. (11):

$$
\begin{gathered}
\tau_{i j}(t+n)=(1-\rho) \cdot \tau_{i j}(t)+\Delta \tau_{i j}(t) \\
\Delta \tau_{i j}(t)=\sum_{k=1}^{m} \Delta \tau_{i j}^{k}(t)
\end{gathered}
$$

here, $\rho$ is the permanence of trail, $0 \leq \rho<1$; 1- $\rho$ is the trail attenuation degree; $\Delta \tau_{i j}(t)$ is pheromone increment on the path $(i, j)$ in this iteration, $\Delta \tau_{i j}(0)=0 ; \Delta \tau_{i j}^{k}(t)$ is the pheromone increment ant $k$ leaves from city $i$ to city $j$ at time $t$, which can be obtained by Eq.(12):

$$
\Delta \tau_{i j}^{k}(t)= \begin{cases}Q / L_{k}, & \text { if } \text { ant } k \text { walk along path }(i, j) \\ 0, & \text { otherwise }\end{cases}
$$


here, $Q$ is pheromone strength, it affects the algorithm convergence speed to some extent; $L_{k}$ is the total length of the path ant $k$ takes in certain iteration.

\section{B. Optimal Distribution Progress Based ACA}

Steps for tobacco optimization distribution using ACA are as follows:

Step 1: parameters initialization, including the coordinates of $n$ tobacco customers, information heuristic factor $\alpha$, relative importance of visibility $\beta$, pheromone strength $Q$ and the maximum number of iterations $N_{\text {cmax }}$;

Step 2: iteration number $N_{\mathrm{c}}$ adds one, that is $N_{\mathrm{c}} \leftarrow N_{\mathrm{c}}+1$;

Step 3: put the initial tobacco customers all the ants locate into $\operatorname{tabu}(k)$;

Step 4: ant $k$ selects the next tobacco customer $j$ to visit according to state transition rules of Eq.(8) and Eq. (9) , and put $j$ into its $t a b u(k)$;

Step 5: check that whether all the tobacco customers are included in the $t a b u(k)$, if yes, goto step 6, otherwise goto step 4

Step 6: record the best route of this iteration, update the pheromone on each path according to Eq.(10), (11) and (12), and empty each ant's tabu table;

Step 7: check whether the maximum number of iterations $N_{\text {cmax }}$ is reached, if yes, goto step 8, otherwise goto step 2;

Step 8: end loop and output the results of the optimal tobacco distribution.

\section{SimUlATION RESUlTS AND ANALYSIS}

In order to verify the validity of ACA to solve battery optimal distribution problem in tobacco distribution network, simulation experiments are carried out compared to the simulated annealing algorithm. The corresponding ACA program is written in $\mathrm{m}$ language.

A tobacco distribution network is taken for an example. Its coordinates are shown in Table 1.

TABLE I. COORDINATES OF TOBACCO CUSTOMER DISTRIBUTION

\begin{tabular}{|c|c|}
\hline Number & Coordinates \\
\hline 1 & $(-7.513 .7)$ \\
\hline 2 & $(-5.514)$ \\
\hline 3 & $(-4.114 .4)$ \\
\hline 4 & $(-2.614 .7)$ \\
\hline 5 & $(-6.512 .9)$ \\
\hline 6 & $(-4.813 .1)$ \\
\hline 7 & $(-3.613 .5)$ \\
\hline 8 & $(-7.711 .5)$ \\
\hline 9 & $(-5.612 .1)$ \\
\hline
\end{tabular}

\begin{tabular}{|c|c|}
\hline Number & Coordinates \\
\hline 10 & $(-6.7$ 10.8) \\
\hline 11 & $(-5.2$ 10.6) \\
\hline 12 & $(-3$ 11.6) \\
\hline 13 & $(-4.211 .8)$ \\
\hline 14 & $(-2.9$ 12.4) \\
\hline 15 & $(-1.8$ 13.2) \\
\hline 16 & $(-3.8$ 10.3) \\
\hline 17 & $(-2.3$ 10.5) \\
\hline 18 & $(-6.19)$ \\
\hline 19 & $(-3.39 .2)$ \\
\hline 20 & $(-7.28)$ \\
\hline 21 & $(-3.98)$ \\
\hline 22 & $(-4.88 .3)$ \\
\hline 23 & $(-28.7)$ \\
\hline 24 & $(-8.37 .5)$ \\
\hline 25 & $(-5.67 .3)$ \\
\hline 26 & $(-86.3)$ \\
\hline 27 & $(-6.86 .5)$ \\
\hline 28 & $(-3.86 .8)$ \\
\hline 29 & $(-1.57)$ \\
\hline 30 & $(-7.15 .3)$ \\
\hline
\end{tabular}

Parameters of ACA are set as follows: $m=50, \alpha=1.5, \beta=2$, $\rho=0.7, Q=8$, Ncmax $=200$.

The optimal tobacco network distribution path using ACA and SA are shown in Figure 1 and Figure 2, respectively.

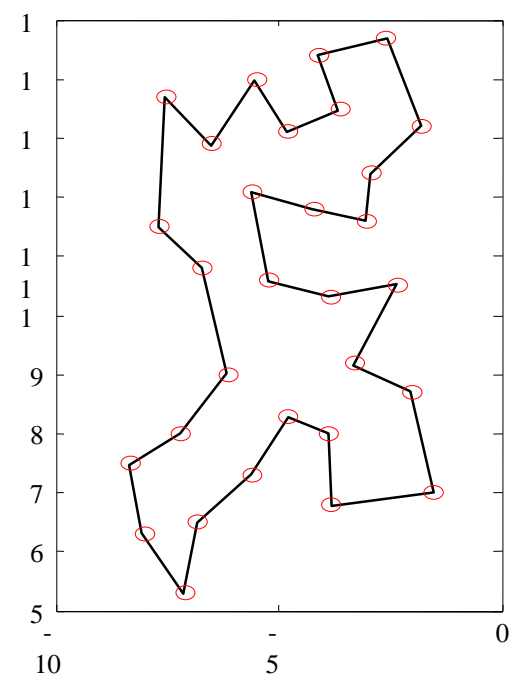

FIGURE I. TOBACCO OPTIMAL DISTRIBUTION PATH USING ACA 


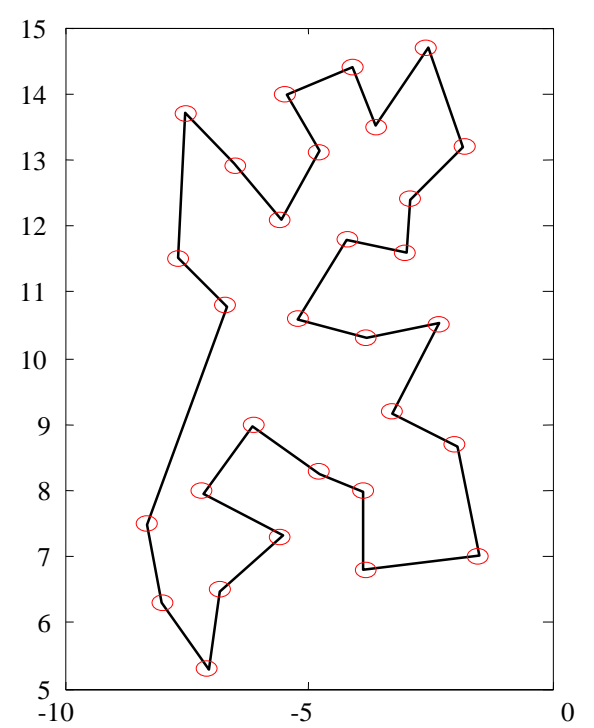

FIGURE II. TOBACCO OPTIMAL DISTRIBUTION PATH USING SA

To see the optimal results more clearly, the shortest distance comparison of battery distribution optimal path using ACA and SA are given in Table 2.

TABLE II. COORDINATES OF TOBACCO CUSTOMER DISTRIBUTION

\begin{tabular}{|c|c|c|}
\hline $\begin{array}{c}\text { Algorithms } \\
\text { name }\end{array}$ & Distance (km) & Improvement (\%) \\
\hline SA & 44.9 & 5.7 \\
\hline ACA & 42.3 & \\
\hline
\end{tabular}

From Table 2, we can see that, the optimal distribution path distances using SA and ACA are $44.9 \mathrm{~km}$ and $42.3 \mathrm{~km}$, respectively. Using ACA would shorten the optimal distribution distance $2.6 \mathrm{~km}$ compared to that of SA. That is to say, the distribution efficiency would improve $5.7 \%$ using ACA.

\section{CONCLUSIONS}

In this paper, we attempt to solve tobacco distribution network optimal distribution problem using Ant Colony Algorithm. Steps for tobacco distribution network tobacco optimal distribution using Ant Colony Algorithm are given out after a mathematical description of vehicle routing optimization problem in tobacco distribution network. The simulation experimental comparison results show its validity.

\section{ACKNOWLEDGMENT}

This research was financially supported by National Natural Science Foundation (NNCF) of China under Grant (61403124) and the Fundation of 2014 Science and Technology project of Henan tobacco companies (2014-22).

\section{REFERENCES}

[1] A. Ye. Optimization of tobacco distribution vehicle routing problem based on genetic algorithm[J]. Application of computer system, 2011,20(4): 241-244.

[2] X. Wang, J. Zhao. Research on tobacco distribution routing problem based on Genetic Algorithm in small and medium sized cities[J]. Logistics technology,2014, 33(3):348-350.

[3] H.Hu, Q.Shi, X. Hu. Fuzzy DEA evaluation model and application of vehicle routing in tobacco distribution center [J]. Journal of Shandong University: Engineering Science Edition,2013,43(3):38-42.

[4] J.Wu. Simulation Research on vehicle routing problem of logistics distribution[J]. computer simulation,2011,28(7):357-360.

[5] Colorni A, Dorigo M, et a1. Distributed optimization by ant colonies. Proceedings of the 1st European Conference on Artificial Life, 1991, 134 l 42. 